Int. J. Dev. Biol. 56: 841-852 (2012)

doi: $10.1387 /$ ijdb.120141ue

\title{
Oocyte ageing and its cellular basis
}

\author{
URSULA EICHENLAUB-RITTER* \\ University of Bielefeld, Faculty of Biology, Institute of Gene Technology/Microbiology, Germany
}

\begin{abstract}
Aneuploidy is extremely high in aged human oocytes. Its cellular origin has been elusive. Trisomy data implicate predominantly meiosis I errors in the genesis of oocyte aneuploidy. Susceptible recombination patterns increase risks for nondisjunction. Cytogenetic analyses of aged human oocytes and embryos from assisted reproduction (ART) suggest that aneuploidy primarily relates to precocious chromatid separation. Oocytes express a spindle assembly checkpoint (SAC), but do not arrest maturation in the presence of improperly attached or single, unattached chromosomes. The SAC may be more permissive by altered gene expression in aged oocytes. Aged oocytes frequently exhibit precocious loss of chromosome cohesion. In experimental models, cohesion cannot be restored once lost, a process possibly occurring during long meiotic arrest. Maternal age, hormonal stimulation, disturbed metabolism, and depletion of the follicle pool contribute to mitochondrial dysfunction, spindle aberrations, and errors in chromosome segregation. Caloric restriction and antioxidants reduce mitochondrial dysfunction and aneuploidy in aged rodent's oocytes. Loss of chromosome cohesion appears to be a major risk factor for aneuploidy by disturbing the sequential separation of homologs and chromatids. A permissive SAC, the presence of risky meiotic exchanges, changes in expression, and failures to resolve improper chromosome attachments, as well as mitochondrial dysfunction may synergistically increase susceptibility to meiotic errors. A healthy life style, mild stimulation and an optimal environment may delay ageing and sustain control over chromosome disjunction, whereas loss of cohesion appears to be irreversible.
\end{abstract}

KEY WORDS: oocyte, ageing, aneuploidy, spindle, chromosome cohesion

\section{Introduction}

Human oocytes are amongst the most long-lived cells in the body. Meiosis is already initiated in the foetal ovary when primary oocytes from oogonial mitotic divisions enter meiotic S-phase and initiate prophase of meiosis I (Fig. 1). For this, the replicated homologous chromosomes each containing two chromatids connected physically by cohesion complexes condense chromatin, pair and recombine with the aid of a synaptonemal complex, a unique meiotic pairing structure (indicated by tripartite red/yellow lines between homologs in Fig. 1) during leptonene, zygotene and pachytene until diplotene stage of prophase I of meiosis. Unlike in male meiosis in which there is a continuous progression from prophase I to completion of first and second meiotic divisions in the testis of the sexually mature male, oocytes become meiotically arrested for long periods of time at prophase/G2 phase of meiosis I (termed dictyate stage), in the human for up to five decades, until they complete meiosis; primordial follicles containing the dictyate stage blocked, prophase I meiotically arrested primary oocytes are formed prior to or shortly after birth from nests of oocytes (nest breakdown), and recruit granulosa cells (Fig. 1). These primordial follicles first have to be recruited into the growing stage, many become atretic and die, and a fraction undergoes an extensive growth phase from the primary to the large antral stage of folliculogenesis (Fig. 1) before the oocytes reach full growth and acquire the competence to resume maturation to metaphase II (Fig. 1, right side) and emit a first polar body (1.PB) containing one set of dyads/metaphase II chromosomes with two sister chromatids (Fig. 1). Since the follicle pool is restricted in size, the recruitment of primordial follicles and follicular atresia eventually lead to ovarian depletion (indicated in Fig. 1), when cycles eventually become irregular and finally pool size reaches a critical size, ovulation seizes and menopause occurs.

Apart from dictyate stage there is a second meiotic arrest in oocytes, in most mammals at metaphase II (MII arrest; Fig. 1). Although chromosomes are aligned and attached to spindle fibres, a condition that leads to anaphase progression in mitosis, meta-

Abbreviations used in this paper: AGE, advanced glycation end product; ART, assisted reproductive technology; SAC, spindle assembly check-point.

\footnotetext{
*Address correspondence to: Ursula Eichenlaub-Ritter. Universität Bielefeld, Fakultät für Biologie Gentechnologie/Mikrobiologie, D-33501 Bielefeld, Germany. Tel: +49-(0)521-106-4832/6907. Fax: +49-(0)521-106-6015. e-mail: EiRi@uni-bielefeld.de
} 
phase II arrest occurs. After fertilization oocytes complete meiosis II, and emit a second, haploid polar body (2.PB; Fig. 1). A haploid male and female pronucleus are subsequently formed followed by first zygotic S-phase in the one-cell embryo. Adult ovaries contain oocyte stem cells that might possess the ability to replenish the ever declining follicle pool (White et al., 2012), but their physiological relevance is currently unclear, and the follicular endowment, continuous recruitment and follicular atresia determine the length of the reproductive period in the human.

The human oocyte is extraordinary susceptible to meiotic errors leading to aneuploidy and autosomal or gonosomal numerical chromosomal aberrations in the zygote and embryo. Aneuploidy is the leading cause of pre- and postimplantation developmental arrest, implantation failure, spontaneous abortion or birth of a chromosomally unbalanced child (e.g. one with three instead of two copies of a chromosomes termed trisomic) (Nagaoka et al., 2012). Thus in assisted reproduction more than $75 \%$ of blastocysts from women over the age of forty are aneuploid (Fragouli and Wells, 2011). Correlations between children with Down syndrome (trisomy 21) and maternal age have been recognized already for many years (Penrose, 1933; Bond and Chandley, 1983; Hassold \& Jacobs, 1984) but the origin of the disturbances causing chromosome nondisjunction in aged oocytes and responsible for reduced fertility and the increased risks for a trisomic 21 or another aneuploid conceptus have remained unknown until recently.
On the subcellular level, it has been postulated that it is mainly the long meiotic arrest that may be causal to age-related aneuploidy and represents the major source of disturbances leading to the oocytes' failures of proper chromosome disjunction (termed nondisjunction). According to the free radical theory of ageing, an accumulation of damage might increase the numbers of dysfunctional organelles, in particular mitochondria (Harman, 1956), due to the prolonged exposure to reactive metabolites and formation of reactive oxygen species (ROS). Low steady-state levels of ROS perform signalling functions to coordinate metabolic and genetic processes but increased ones can damage cells. ROS and advanced glycation end products (AGEs) from metabolism that fail to become immediately detoxified can contribute to adduct formation on lipids, proteins and DNA and induce rises in mutations in mitochondrial and nuclear DNA (for references see: Eichenlaub-Ritter et al., 2011; Tatone et al., 2011). Reduced local supply of high energy substrates like ATP by mitochondria can affect spindle formation and regulation in the aged oocyte resuming maturation, such that aberrant spindles become a hallmark of aged oocytes (e.g. Battaglia et al., 1986).

Apart from chronological age and length of the arrest period, the depletion of the primordial follicles in the ovary may be most critical by altering the follicular and oocytes' microenvironment and hormonal homeostasis (Brook et al., 1984). Within the ovary and follicle, a reduced oxygen supply by insufficient vascularisation (Gaulden, 1989), and altered hormonal homeostasis (e.g. elevated

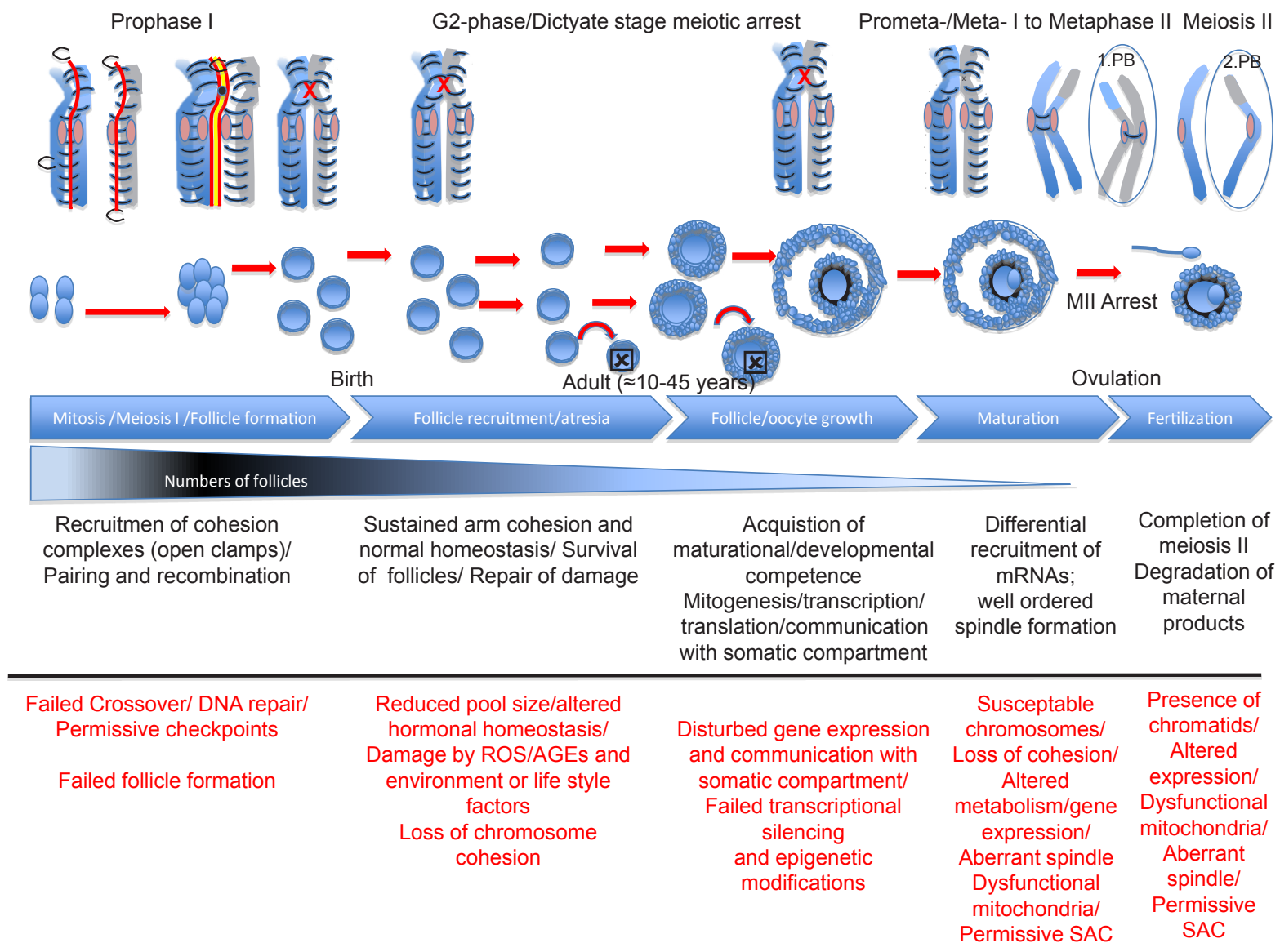

Fig. 1. Oocyte and follicle development, meiotic stages, pool size, key events in development and suggested disturbances contributing to age-related meiotic errors. Black lettering: normal development; red lettering: disturbances related to oocyte ageing and suscepibility to meiotic errors. For further explanation, see text. 
follicle stimulating hormone, FSH) might cause compromised interactions and suboptimal paracrine and autocrine signalling within the follicle and between the aged oocyte, the somatic compartment and the neuro-endocrine axis thereby increasing the risks for errors in chromosome segregation. The genetic background affecting the initial formation/survival of oocytes and follicles before and after birth in addition to exposures to toxicants e.g. to endocrine disrupting chemicals or contaminants in drinking water, can affect health and survival of oocytes and follicles (Susiarjo et al., 2007; Pacchierotti et al., 2007). Finally, life style factors and pathologies may play a role in affecting mitochondrial function/metabolism (e.g. diabetes) (Wang et al., 2009) that ultimately can result in oocyte ageing, reduced oocyte quality, aneuploidy and premature ovarian insufficiency (POI).

Since chromosomes appear differentially susceptible to agerelated nondisjunction (reviewed by Hassold and Hunt, 2001; Nagaoka et al., 2012) it has been postulated that events in the foetal ovary are of relevance. At prophase I stage, chromosomes begin to pair and recombine in the foetal ovary (Fig. 1). Oocytes resting within primordial follicles may contain specific 'risky' recombination patterns that will predispose oocytes ovulated late in the reproductive life to random chromosome segregation, particularly at meiosis I.

In order to discriminate between influences of the chronological maternal age per se (reflected in the length of the meiotic arrest), the size of the follicle pool (reflected in markers like anti-muellerian hormone, $\mathrm{AMH}$ ), and the hormonal status (e.g. increases in follicle stimulating hormone, $\mathrm{FSH}$ ), incidence of trisomic conceptions and abortions have been analysed in retrospective and prospective studies. For instance, women with a trisomy 21 pregnancy at a younger age have often lower AMH levels, suggesting a correlation between aneuploid oocytes and a limited ovarian reserve (van der Stroom et al., 2011). Accordingly, younger poor responding patients (.36 years) who had presumably a reduced ovarian reserve, had a statistically significant increased miscarriage rate, possibly related to an increased aneuploidy rate, compared to normal responders (Haadsma et al., 2010). However, elevated FSH but not AMH related to trisomic conceptions in another study (Kline et al., 2011) suggesting that hormonal imbalance may influence chromosome segregation, for instance, by directly affecting maturing oocytes (Xu et al., 2011). In a mouse model, unilateral ovariectomy resulted in increased numbers of spontaneously ovulated metaphase II oocytes with unaligned chromosomes in the middle-aged females with depleted follicle pools, unlike age-matched controls but similar to the advanced aged spontaneously ovulated control oocytes. The pole-to-pole distance of the metaphase II spindle related to chronological age of the mother instead of pool size, suggesting that both, age and follicle depletion, contribute to risks for meiotic errors (Eichenlaub-Ritter et al., 1988). A recent meta-analysis of 22 genome-wide association studies on natural age at menopause in humans identifed genes that may contribute to rapid follicle depletion and reduced pool size (Stolk et al., 2012). Such approaches can help to identify genes associated with increased risks for errors in chromosome segregation in aged oocytes that may be correlated to reduced pool size.

There may be also a link to altered metabolism in age-related nondisjunction. For instance, older animals in many laboratory strains of mice have an increased body weight and are obese. In oocytes of the CD1 strain this appears associated with spindle defects, higher serum cholesterol, abnormal glucose tolerance and lower levels of circulating AMH hormone compared to the younger and leaner controls (Hirshfeld-Cytron et al., 2011). At the cellular basis it has been noticed that spindle aberrations become also increased in aged human oocytes (e.g. Battaglia et al., 1986) and thereby are a hallmark of reproductive ageing. This might relate to altered gene expression, e.g. of spindle, and cell cycle regulating factors (see below).

Furthermore, several studies imply that the kinetics of oocyte maturation become perturbed, either shortened or protracted (e.g. Eichenlaub-Ritter and Boll, 1989; Volarcik et al., 1998), suggesting links of increased aneuploidy to loss of cell cycle control. Furthermore, chromosomes frequently fail to properly align at the spindle equator in aged oocytes (termed congression failure), and this has been correlated to spindle aberrations as well as altered expression patterns and cell cycle control (e.g. Pan et al., 2005). Ultimately it may be that chromosomes that lack recombination and chiasmata or behave as ,functional' univalents due to loss of chromosome cohesion cannot be properly attached to the spindle, orient to opposite spindle poles, and are likely to randomly segregate and increase oocyte aneuploidy (see below).

In the following the lesions and events contributing to oocyte ageing and increases in meiotic errors at the cellular basis will be discussed in more depth.

\section{Recombination rate, and meiosis I vs. meiosis II errors in human oocytes}

While aneuploidy is rare in human sperm (about 1-2\% on average in sperm of healthy males) about $20 \%$ of all human oocytes over all maternal ages carry a numerical chromosomal aberrations (Hassold et al., 2007). Polar body analysis by array comparative genomic hybridization to assess imbalance in all chromosomes show that oocyte aneuploidy increases to rates of $50 \%$ or more in oocytes of women of advanced age (e.g. Fragouli et al., 2011; Handyside et al., 2012). After fertilization many aneuploidies will give rise to developmental arrest, and implantation failure of the embryo (recently discussed by Fragouli and Wells, 2011; Nagaoka et al., 2012). Surviving autosomal aneuploidies are likely to induce spontaneous abortion and stillbirth, and only a minority will be able to develop further and cause aneuploidy and trisomy like Down syndrome in life births (Nagaoka et al., 2012). Maternal age and oocyte aneuploidy are thus the predominant cause of reduced fertility in aged women and low success rates in ART.

In the past, presence of polymorphisms at or close to the centromere and along chromosome arms of extra chromosomes in trisomies have been used to trace the origin of chromosomal errors to either maternal (indicated by grey chromatids) or paternal (indicated by blue chromatids in Fig. 2) meiosis I or meiosis II, or a postzygotic mitotic error in chromosome segregation. It appears that the majority of errors giving rise to the common trisomies in naturally conceived offspring in humans involve chromatids of both maternal homologs and were therefore initially believed to be derived by a first meiotic nondisjunction event in oogenesis (reviewed by Hassold and Hunt, 2000). If both homologs fail to divide at meiosis I but both of their sister chromatids of each homolog segregate to the second polar body, a sister chromatid of each homolog would be retained in the oocyte. In fact, $76 \%, 100 \%$, $65 \%$ and $94 \%$ of extra chromosomes in trisomies 15, 16, 21 and 
22, respectively, appear associated with two maternally-derived chromosomes that come from both maternal homologs except at sites at which recombination occurred. Trisomy 18 is an exception as $58 \%$ of cases involve nondisjunction of chromatids. The trisomy 18 cases contain two copies of maternal chromosomes with identical centromeres and alleles of one homolog except for sites of recombination. Only 33\% are meiosis I errors apart from $11 \%$ postzygotic mitotic errors in trisomy 18 (Hassold et al., 2007). These findings already suggest that susceptibility to meiotic errors at younger or advanced maternal ages is chromosome specific and potentially involves differences in length of the chromosome and distribution of exchanges on individual bivalents. This can be also deduced from the age-related increase in the incidence of trisomies. For instance, the risk for trisomy 16 increases over all maternal ages while that for trisomy 15 or 21 is comparatively low at younger ages but increases exponentially from about 35 years of age (reviewed by Nagaoka et al., 2012).

Detailed analysis of the recombinational history of extra chromosomes more recently also suggest particular risks depending on exchange patterns, for instance, in large cohorts of trisomy 21 cases and age-matched euploid controls (Oliver et al., 2008). Thus, a large percentage (about 40\%) of the errors in trisomy 21 involve achiasmatic chromosomes that lack an exchange. The absence of recombination appears therefore to predispose oocytes to meiotic errors of this and other chromosomes at all maternal ages. Moreover, the recombination map of extra maternally derived chromosomes 21 involving nondisjunction of homologous chromosomes (meiosis I errors) in oocytes of younger mothers appears shorter compared to the normally segregating chromosomes. Frequently only a single exchange close to the distal part of the chromosome, in the proximity of the telomere has been detected. Amazingly, extra chromosomes in trisomy 21 derived from a maternal first meiotic error in the aged oocytes appear to have a very similar recombination map as those segregating normally and leading to a euploid child. Therefore, it appears that a single distal exchange is risky, but even those chromosomes 21 with a normal exchange pattern become susceptible to a first meiotic error within an aged ooplasm. Finally, those trisomies 21 involving chromatids of one homolog and presumably derived by a second meiotic error had a longer recombination map compared to the normally segregating chromosome 21 (Oliver et al., 2008). According to this, chromosomes 21 with an excess of recombination in the proximal, pericentromeric part of the chromosome appear highly susceptible to fail to separate chromatids in meiosis II, particularly in an aged ooplasm. Since chiasmata usually resolve at meiosis I, an event at first meiosis must influence behaviour of chromosomes at anaphase II. For instance, a failure to separate the two homologs that are connected by a pericentromeric exchange at anaphase I may cause that the two homologs remain in the ooplasm and disjoin reductionally and with a delay at meiosis II instead of separating their chromatids at second meiotic division. Accordingly, the first polar body would not contain this chromosome while two chromatids of one homolog of a bivalent would remain in the oocyte and embryo and two also in the second polar body. This mimics a classical second meiotic error in which the two chromatids derived from a normal first meiosis fail to disjoin at anaphase II.

Since different trisomies have different susceptibilities to meiosis I or II errors, and distribution of exchanges appears differentially related to risks for first or second meiotic nondisjunction event in young or aged oocytes, it was postulated that more than one event affects ordered chromosome segregation at oogenesis at the cellular level. Absence or increases in steric constrains of the orientation and attachment of centromeres to spindle fibres to opposite poles in certain types of distribution and numbers of ex-

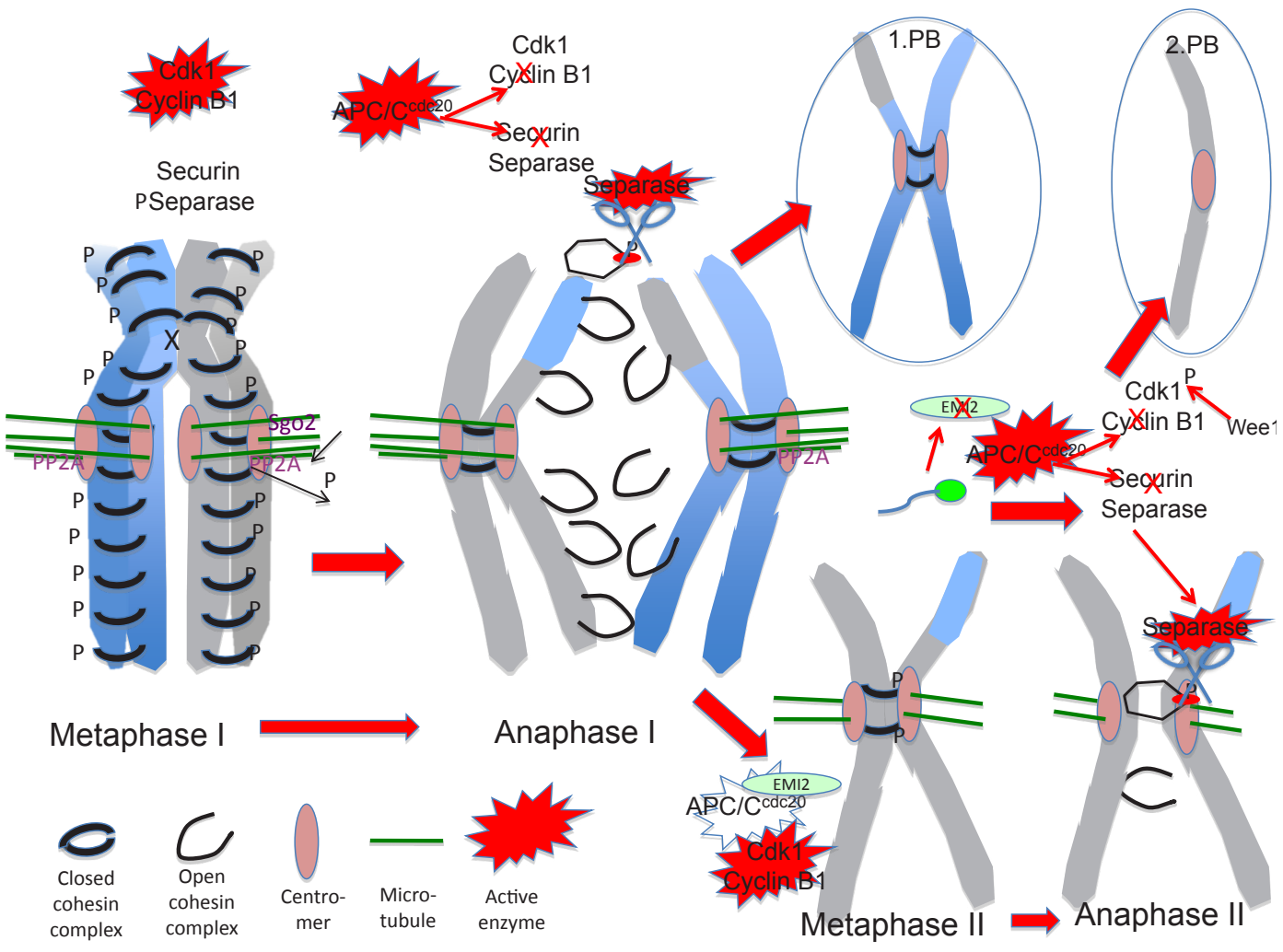

Fig. 2. Oocyte maturation and chromosome segregation at anaphase I and anaphase II by activity of $\mathrm{APC} / \mathrm{C}^{\mathrm{cdc} 20}$ recognizing and causing degradation of cyclin B1 and securin. Release and activation of separase causing proteolysis of phosphorylated (p) cohesin and opening of cohesin complex. Prevention of phosphorylation by SGO2 and PP2A recruitment to centromeres, and activity of centromeric PP2A at meiosis l; fertilization induced destruction of EMI2 inhibitor of APC/Ccdc20 and progression to anaphase II; formation of first polar body (1.PB) containing one homolog with two sister chromatids of each autosome as a result of normal anaphase I, and formation of the second polar body $(2$. $P B)$ containing one chromatid of each autosome after completion of anaphase II, respectively. For further explanation, see text. 
changes on each chromosome may pose a risk over all ages (e.g. one distal exchange in the short, acrocentric chromosome 21) or preferentially increase risks in aged ooplasm (e.g. a pericentromeric exchange of chromosome 21). As an example, chromosomes 15 with 3-4 exchanges contribute to meiosis I errors and trisomy 15 in oocytes of aged mothers in about $40 \%$ of trisomy 15 cases but not in those from younger mothers (Robinson et al., 1998).

Recombination rates and distribution of crossovers differ greatly between individuals and between male and female meiosis. While in male meiosis distal exchanges prevail, the overall recombination map is longer and there tend to be more interstitial exchanges in oogenesis compared to spermatogenesis (e.g. Cheng et al., 2009). Thus it appears that gender differences in recombination rates and pattern that relate also to susceptibility to meiotic errors result from differences in the regulation of female and male meiosis. A master regulator influencing hot spots of recombination and crossover has only recently been identified (Baudat et al., 2010; Grey et al., 2011). One variant of a gene controlling recombination rate affects male meiosis differently from female meiosis (Kong et al., 2008). Rodent models suggest that there are common mechanisms but also sexual dimorphism in activity of gene products involved in pairing, recombination and DNA repair as well as in checkpoint controls causing meiotic arrest between male and female meiosis (Hunt and Hassold, 2002). For instance, failures to form the synaptonemal complex in mice deficient in Hormad1 component of the synaptonemal complex cause pachytene arrest in spermatogenesis, while oocytes more likely progress to meiosis I and metaphase II. Embryogenesis arrests at blastocyst stage presumably because of oocyte-derived aneuploidy (Shin et al., 2011). Oocytes are also more likely to escape a meiotic block e.g. in response to a deficiency in genes in DNA repair (e.g. $\mathrm{mh} / 1$ ) and become susceptible to aneuploidy by presence of univalents (Nagaoka et al., 2011). The differences in checkpoint control also contribute to gender-specific transmission bias of reciprocal translocations and sterility since they are more frequently causing meiotic arrest in male compared to female meiosis (reviewed by Kurahashi et al., 2012). The synaptonemal complex has been studied with respect to the distribution of recombination nodules (dark dots in Fig. 1) enriched in recombination and DNA repair enzymes (e.g. the Mh/1 protein). These mark sites of exchanges. Alarge percentage (about $10 \%$ ) of human oocytes contained one or several crossover-less chromosomes while achiasmatic chromosomes are rare in spermatogenesis (Cheng et al., 2011). When oocytes with achiasmate chromosomes (Fig. 3D) escape checkpoint surveillance, they can contain univalents after resumption of maturation that predispose to random segregation and aneuploidy.

Hendersen and Edwards (1968) postulated that recombination rates differ between young and aged oocytes. They proposed that oocytes that are matured and ovulated late in the reproductive period (aged oocytes) are derived from oogonia and oocytes that initiate meiosis late in the foetal ovary and are recombination deficient. Provided they become recruited in the order they are formed (according to a "production line"), these "delayed" and aged oocytes would possess high numbers of univalents which may randomly segregate. This "production line hypothesis" has been tested by analysis of recombination frequency of maternally- and paternally-derived chromosomes in children of aged mothers with euploid constitution. Increased rather than decreased recombination is characteristic for chromosomes of euploid children from aged mothers in a European cohort (Kong et al., 2004) suggesting that high recombination rates protect aged oocytes from nondisjunction and thereby promote survival of embryos. A recent study on a large number of polymorphic markers in euploid children in a Canadian cohort found a slight reduction in the average recombination frequency of maternally- but not paternally-derived chromosomes of euploid children of up to 32 year old mothers but not in children from older women, inconsistent with the prediction by the "production line" (Hussin et al., 2011). Therefore, even tough a production line may exist it is unlikely to dramatically affect exchange rates on chromosomes and thereby increase risks for meiotic errors in aged oocytes.

Studies on aneuploidies involving the most common trisomies by fluorescence in situ hybridization (FISH), classical spreading and banding approaches as well as recent analyses of oocytes and polar bodies using array comparative genomic hybridization (aCGH) show that numerical chromosomal aberrations most frequently involve chromatids and not whole chromosomes (homologs)- unlike expected from the trisomy data (e.g. Pellestor et al., 2004; Magli et al., 2012; Fragouli et al., 2011; Handyside et al., 2012; Gabriel et al., 2011). Chromatid type errors frequently involve multiple chromosomes in the aged but less likely in the younger oocytes (Handyside et al., 2012). On first view, these observations appear to contradict data from trisomy studies suggesting that mainly first meiotic errors give rise to numerical aberrations in the oocyte and embryo. However, it is conceivable that loss of chromosome cohesion may play a role, such that errors in separation of chromatids occur at meiosis I and II and present the primary predisposing factor.

\section{Regulation of differential loss of chromosome cohesion at meiosis and evidence that loss of cohesion can be causal to errors in chromosome segregation in aged oocytes}

Chromosome segregation is tightly regulated in mitosis. Anaphase progression usually occurs only when all chromosomes have attached to spindle fibres at their kinetochores, specialised structures at centromeres containing DNA binding and centromere proteins (CENPs) and regulatory components. The latter sense attachment and tension from pulling forces by spindle microtubules by attachment of sister chromatid centromeres to opposite spindle poles (reviewed by Musacchio, 2011). At prometaphase spindle microtubules are metastable and there is rapid polymerisation/ depolymerisation involving microtubule associated and motor proteins as well as activity of depolymerases e.g. of the kinesin 13 family of motor proteins (Ems-McClung and Walczak, 2010). At late prometaphase to metaphase transition dynamic microtubule turnover is altered and the spindle assembly checkpoint (SAC) becomes activated.

The SAC represents a surveillance mechanism ensuring that only those cells that have properly attached chromosomes (unusually when they are congressed and aligned at the spindle equator) will go into anaphase (reviewed by Musacchio, 2011). The silencing of the SAC is a prerequisite to anaphase progression. By sensing unattached chromosomes that accordingly are often not properly aligned and are lacking tension from bi-polar attachments, a conformational change in a checkpoint protein is induced. This leads to the formation of a "mitotic checkpoint complex" (MCC) producing a "wait for anaphase" signal. The cur- 
rent model predicts that MCC (containing the Mad2 checkpoint protein in open/closed dimerized form) binds to the co-activation factor of the anaphase promoting factor/cyclosome (APC/C), the Cdc20 (cell division cycle 20) protein. This prevents activation of the APC/C and the targeting of substrates of the APC/C by the $\mathrm{Cdc} 20$ protein. APC/C is a ubiquitin ligase complex that mediates polyubiquinylation of proteins marking them for degradation by the proteasome. In oogenesis two co-activators of APC/C are involved in cell cycle progression: the APC/ ${ }^{\text {cdh } 1}$ regulates the G2-phase meiotic arrest and resumption of maturation, and later after GVBD prevents a precocious increase in the $\mathrm{Cdc} 20$ protein and $A P C / C^{c d c 20}$ activation (reviewed by Homer, 2011). The APC/C ${ }^{\text {cdc20 }}$ recognizes motifs on target proteins like Cyclin B1, the regulatory subunit of Cdk1/CyclinB1 (also termed MPF, maturation promoting factor), the master regulator of M-phase and on securin (see below).

A signal from a single unattached chromosome is sufficient to prevent anaphase progression in mitotically dividing cells. When all chromosomes are attached and there is no more conformational change of Mad2 checkpoint protein the latter appears to become transported away from centromeres along spindle fibres towards the spindle poles by dynein microtubule motor proteins. Therefore, the SAC is silenced or satisfied, and the APC/C ${ }^{\text {cdc20 may become }}$ activated when no more MCC is produced (Musacchio, 2011).

The securin protein is present in a complex with separase, a proteolytic enzyme, prior to anaphase. Separase is inactive in the complex with securin and by inhibitory phosphorylations by the MPF/Cdk1 kinase. In case APC/C ${ }^{\operatorname{cdc} 20}$ becomes activated, it triggers securin degradation, and causes the release and activation of separase. In turn, the proteolytic separase can cleave proteins in the chromosome glue holding sister centromeres together at M-phase of mitotic division (cohesin complex), such that the chromatids can separate from each other (reviewed by Vogt et al., 2008). The cohesin complex contains highly conserved proteins of the structural maintenance of chromosomes (SMC) family that are believed to embrace the chromatids like a ring or a clamp (reviewed by Nasmyth, 2011). Closure of the clamp is by binding to conserved proteins of the kleisin family of proteins like Scc1 in mitosis and accessory proteins (Sa1/Sa2). Loading and stabilization of the cohesin complex involve different accessory factors and posttranslational modifications while opening in M-phase to anaphase transition occurs by proteolytic cleavage of the kleisin component (in mitosis Scc1, in meiosis phosphorylated Rec8). Some cohesin proteins are expressed in all dividing cells while others are mitosis or meiosis-specific and their unique roles are not yet fully understood (reviewed by Nasmyth, 2011).

In mitosis cohesion on chromosome arms is initially present all along the chromatid arms and centromeres. Cohesion becomes lost along the sister chromatid arms at late prometaphase by nonproteolytic events involving protein phosphorylation of a mitotic cohesin protein. Cohesion at centromeres is retained involving activity of proteins like shugoshin (the Japanese name for "guardian spirit"), Aurora kinase B and phosphatase PP2A that prevent critical protein phosphorylation of the kleisin cohesins (Ishiguro et al., 2010; Tanno et al., 2010). Cohesion at centromeres is only lost after the SAC is satisfied, and no more MCC is produced. APC/ $\mathrm{C}^{\text {cdc20 }}$ becomes activated, and the separase is released from securin protein after securin's APC/C induced proteolytic degradation. In a next step, active separase can cleave the cohesin Scc1 protein in the cohesin complex in mitosis. Upon this, the ring-like complex of cohesin proteins opens and releases the two centromeres of the sister chromatids to separate from each other at anaphase.

In contrast to mitosis, meiotic division comprises two consecutive M-phases without intervening S-phase and control over anaphase I and II progression is much more complex (Fig. 2). A reductional separation of homologs at anaphase I results in separation of the two homologs each of which consists of two sister chromatids. This is followed by an equational separation of chromatids attached to opposite spindle poles (amphitelic) (Fig. 2) at anaphase II that resembles mitotic chromosome separation. The cohesin complexes mediating physical attachment between sister chromatids are recruited in S-phase of meiotic division (indicated by open clamps in left part of Fig. 1). Recruitment of cohesins already occurs in the foetal ovary in oogenesis, when primary oocytes undergo S-phase. Accordingly, when recombination occurs between the two chromatids of the parental homologs, the exchanges and the cohesion between sister chromatids in individual homologs physically connect the two homologous chromosomes within the bivalent up to metaphase II (Fig. 1). Importantly, cohesion has to be maintained from prophase I in the primary oocyte of the foetal ovary throughout meiotic arrest, in humans for decades, until oocytes resume meiosis and undergo meiotic divisions.

Unlike in mitosis, sister chromatid arm cohesion persists until anaphase I when activation of the APC/C ${ }^{\text {cdc20 }}$ induces securin degradation, and separase activation (Fig. 2). Separase recognises a meiotic kleisin cohesin protein, Rec8 but only when Rec8 is phosphorylated (indicated by $P$ in Fig. 2). This is at the sister chromatid arms in meiosis I. Cleavage by separase causes loss of arm cohesion and thereby chiasma resolution. The Rec8 protein at sister centromeres remains intact and centromeres attached since phosphorylation of Rec8 is prevented by the activity of shugoshins and phosphatase PP2A. Shugoshins appear necessary to bind and retain PP2A at centromeres in oogenesis and prevent precocious chromatid separation in mouse and human oocytes (e.g. Ishiguro et al., 2010; Garcia-Cruiz et al., 2010). The targeting of shugoshin itself that is necessary to recruit PP2A to the centromeres is involving Aurora kinase $B$ and causes also recruitment of mitotic centromere associated kinase (MCAK) to centromeres, which is a depolymerase resolving improper microtubule attachments (Tanno et al., 2010).

In case arm cohesion between sister chromatids is lost precociously prior to anaphase I chiasmata are resolved untimely. Homologs then behave as functional univalents (Fig. 3B). These are at risk to attach randomly to one spindle pole (Fig. 3B) or improperly to opposite spindle poles (see below, Fig. 3 C,D) and predispose oocytes to aneuploidy (Kouznetsova et al., 2010). Presence of univalents in male meiosis leads to meiotic arrest while a single or several univalents do not cause a block in oocyte maturation, as for instance shown in monosomy $X$ oocytes in the mouse (LeMaireAdkins et al., 1997) or in mouse oocytes deficient in a recombination enzyme (in Mlh1 knockout females; Nagaoka et al., 2012). The unique insensitivity of oogenesis to the presence of univalents can be explained by the unique behaviour of centromeres of sister chromatids in the univalents. Frequently centromeres of sister chromatids attach to opposite (amphitelic) instead of only one spindle pole (syntelic) as should occur in first meiosis (Fig. 3 C,D) (Kuznetsova et al., 2010). Accordingly, sister chromatids segregate equationally instead of reductionally at anaphase I of first meiotic division. The attachment to opposite spindle poles 
presumably contributes to the permissive checkpoint control as the SAC does not appear to monitor tension but rather centromere attachment in female meiosis (Gui and Homer, 2012; Kolano et al., 2012; Lane et al., 2012; Sebestova et al., 2012).

When cohesion between sister centromeres is not only lost precociously at chromosome arms but also at centromeres (Fig. 3I), random segregation of the sister chromatids may occur at anaphase I, such that single or several chromatids become distributed to the first polar body or remain in the oocyte. In case, loss of cohesion is delayed this may induce chromosome lagging on the spindle, and random segregation of bivalents to oocyte or first polar body (true ,nondisjunction'). The mode of behaviour of meiotic chromosomes with and without cohesion is schematically depicted in Fig. 3, demonstrating that loss of cohesion can cause errors and random segregation of homologs or chromatids. In normal meiotic divisions, the cohesion of centromeres of sister chromatids in meiosis II facilitates bi-polar attachment, similar to mitosis (Fig. 3H). Upon fertilization, phosporylated Rec8 at centromeres is recognized by separase, and becomes cleaved (Fig. 2). In this way physical attachment between sister chromatids is lost such that they can separate at anaphase II (reviewed by Vogt et al., 2008). Accordingly in normal meiosis the first polar body should contain homologs with two sister chromatids and the second polar body chromatids (Fig. 2).

In metaphase II arrested oocytes the SAC is satisfied. Some $\mathrm{APC} / \mathrm{C}^{\mathrm{cdc} 20}$ is activated and transient cyclin $\mathrm{B} 1$ degradation occurs. Cyclin B1 can be replaced from cytoplasmic stores requiring protein synthesis. A block in translation of metaphase II arrested oocytes or extended oocyte postovulaory ageing can thus induce transient loss of cohesion between sister chromatid centromeres (Sowarto et al., 1995; Mailhes et al., 1998). However, usually oocytes remain meiotically arrested because the full activation of the APC/ $\mathrm{C}^{\mathrm{cdc} 20}$ is prevented by binding of its Cdc20 co-activator to EMI2 (early mitotic inhibitor 2 ) protein. The rise in intracellular calcium by fertilization activates a calcium-dependent calmodulin kinase II. The latter triggers phosphorylation of EMI2 and its degradation such that APC/C ${ }^{\text {cdc20 }}$ becomes activated, and induces polyubiquinylation and degradation of cyclin B 1 and securin (Fig. 2). The calcium-calmodulin dependent kinase II also causes activation of Wee1B, a kinase that puts an inhibitory phosphorylation on Cdk1 thereby inactivating MPF. This contributes to rapid inactivation of the Cdk1(Oh et al., 2011). Active separase can recognize and proteolytically cleave the phosphorylated Rec8 at the sister centromeres such that chromatids can disjoin and become segregated to the oocyte and the second polar body.

Several recent reports from experimental models, mainly in ro-
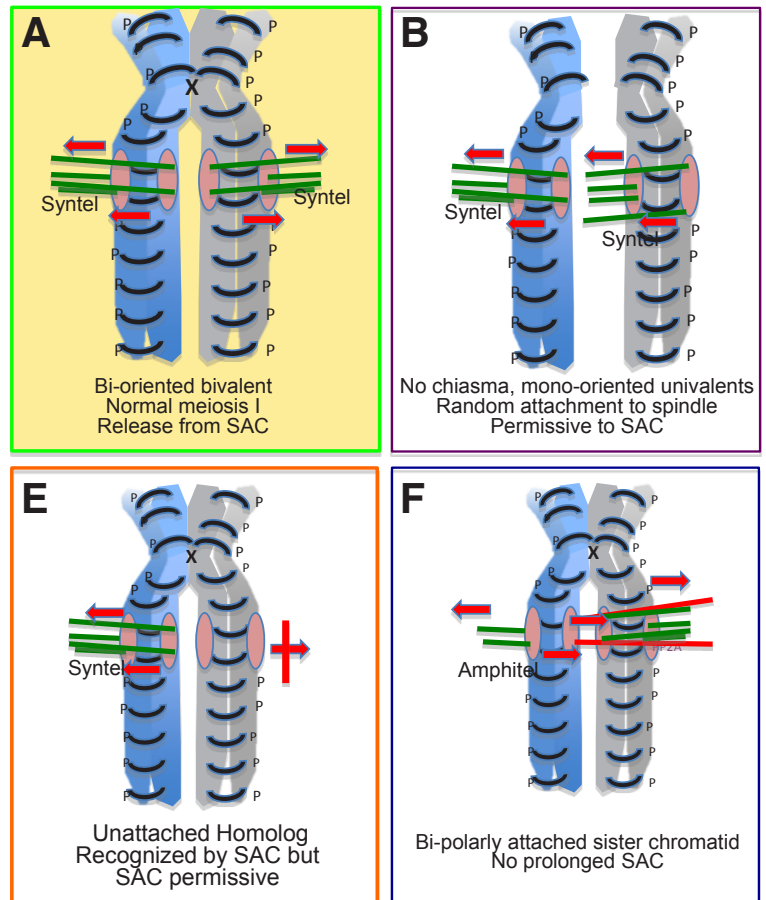
Bi-polarly attached sister chromatid
No prolonged SAC
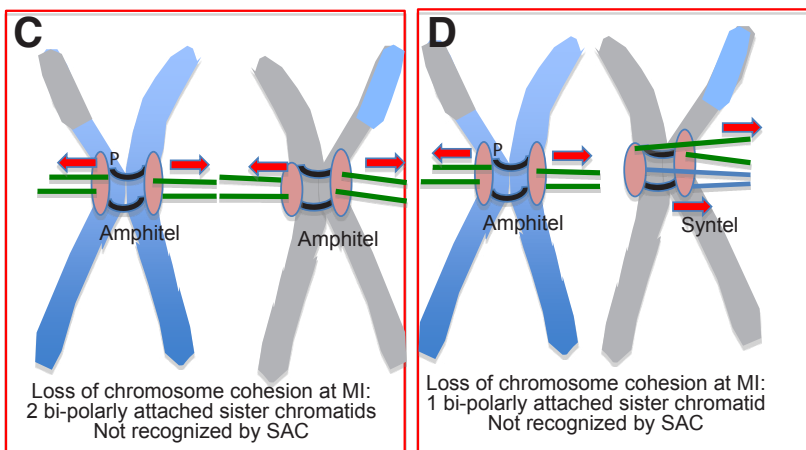

G
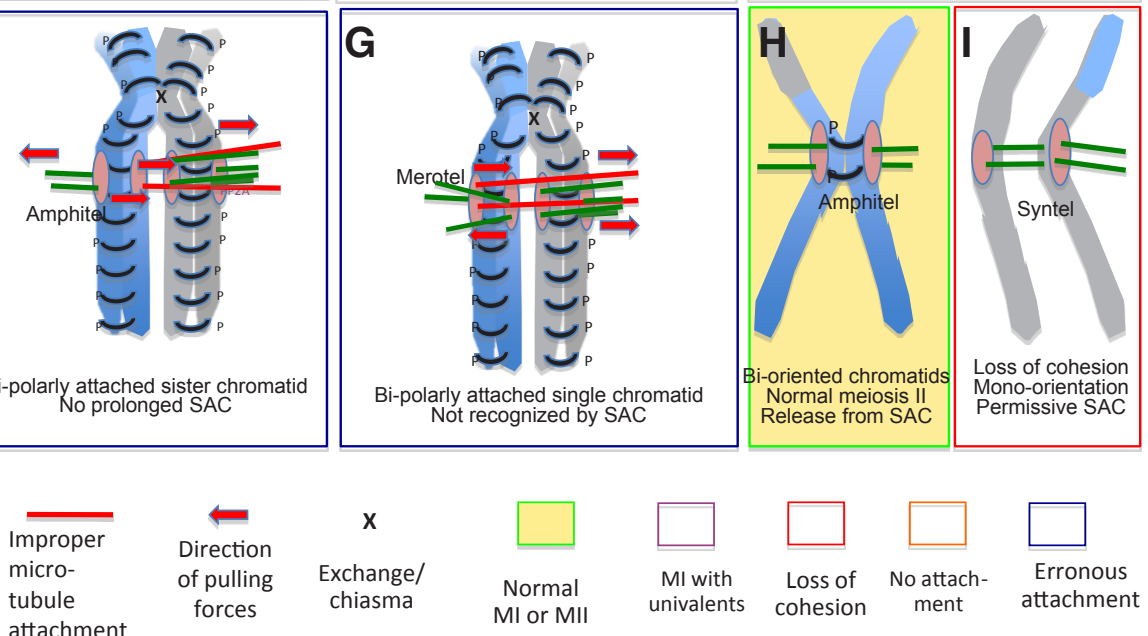

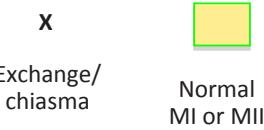

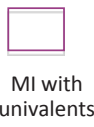

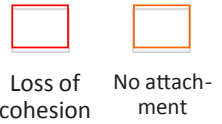

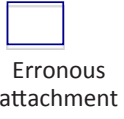

Fig. 3. Attachment and orientation of chromosomes at meiosis I and meiosis II, and recognition by the spindle assembly check-point (SAC). Normal meiosis I with syntel attachment of homologs (A) and normal meiosis II with amphitel attachment of sister chromatids (H); random attachment of achiasmatic, univalent chromosomes to one spindle pole (B); loss of cohesion resulting in bi-polar attachment and precocious separation of sister chromatids at anaphase I (C,D); failures in proper chromosome attachment resulting in meiotic errors when they are not recognized by the SAC or corrected by depolymerase activities in time prior to anaphase / (E-G); random attachment of sister chromatids to same spindle pole after precocious loss of cohesion at centromeres (I). For further explanation, see text. 
dent, suggest that precocious loss of cohesion may be one primary cause of predisposition to errors in chromosome segregation of aged oocytes. In mice lacking a meiotic cohesin protein, Smc1 $\beta$, recombination is disturbed, and there is an overall increase in univalents and bivalents with unusual exchange patterns and aneuploidy (Hodges et al., 2005). Aneuploidy rises also earlier compared to controls when females age (Hodges et al., 2005). Aged oocytes of some strains of mice produce not only more aneuploid oocytes compared to younger females but are also characterised by depleted cohesin and shugoshin protein at centromeres in their aged oocytes (Lister et al., 2010). Loss of attachment at sister centromeres appears especially high when aged mouse oocytes are from stimulated cycles and/or matured in vitro to metaphase II (Chiang et al., 2010; Lister et al., 2010; Merriman et al., 2012). Knockdown of Rec8 expression in oocytes of the growing follicle pool does not induce loss of chromosome cohesion suggesting de novo translation past dictyate arrest is not necessary to maintain cohesion in oocytes (Revenkova et al., 2010). However, depletion of younger mouse oocytes of the Rec8 cohesin protein by targeted proteolysis causes loss of chromosome cohesion. The rises in aneuploidy cannot be compensated/reversed by overexpression of a functional non-cleavable human Rec8 protein prior to and at meiotic maturation (Tachibana-Konwalski et al., 2010). Therefore, it appears that losses of cohesion cannot be replaced past early prophase I and are relevant for ageing in oocytes.

It is feasible that under physiological conditions gradual loss of cohesin takes place during the long meiotic arrest period. Accordingly, hyperactive separase induces faster resolution of chromosome cohesion in aged compared to young oocytes in an experimental mouse model (Chiang et al., 2011). In humans meiotic arrest is much longer compared to most animals like the mouse which could explain some species-specific differences in susceptibility to meiotic errors and also the high susceptibility of shorter chromosomes. Loss of cohesion might be due to hydrolysis, transient low activity of separase, or by an imbalance of factors stabilizing or releasing cohesion. Histone deacetylase has been implicated in deacetylation of a cohesin protein, Smc3, that can induce cohesin dissociation (for review see Nasmyth, 2011). Whether acetyl transferases or deacetylates contribute to loss of cohesion in aged oocytes is currently unknown.

Since there is also evidence from the mouse model that hormonal stimulation can affect cohesion between sister chromatid centromeres (Merriman et al., 2012), it remains to be determined whether it is mainly chronological age or also unfavourable microenvironment at oocyte growth and maturation, in spontaneous or stimulated cycles, or exposures and intrinsic degenerative processes throughout reproductive life that influence loss of chromosome cohesion in human oocytes. Culture conditions during resumption of maturation in vitro in cumulus denuded mouse oocytes causes precocious loss of cohesion at sister centromeres prior to metaphase II (Cukurcam et al., 2007). It thereby predisposes to second meiotic errors. Presence of cumulus providing metabolites from glycolysis and follicular fluid meiosis activating sterol (FF-MAS), a sterol intermediate in the cholesterol biosynthetic pathway produced within the follicle in vivo reduced or prevented the incidence of precocious chromatid segregation prior to anaphase II in this model (Cukurcam et al., 2007). Although human oocytes matured with FF-MAS did not have reduced aneuploidy rates compared to in vivo ovulated ones, FF-MAS might have protected oocytes from errors in chromosome segregation induced by the in vitro maturation conditions (Smitz et al., 2007). From these observations it appears that multiple factors may influence fidelity of chromosome segregation in young or aged oocytes. For instance, maternal age and hormonal stimulation increased the distance between sister centromeres in metaphase II arrested mouse oocytes synergistically (Merriman et al., 2012).

So far there is no conclusive evidence that aged human oocytes possess reduced cohesion protein (Garcia-Cruiz etal., 2011). However one report showed univalent 16 chromosomes in aged human oocytes (Angell, 1997). Since chromosome 16 contains usually at least one chiasma this observation supports the concept of loss of cohesion as major source of errors in chromosome segregation. The inter-kinetochore distance was also increased in aged, in vitro matured human oocytes compared to the distance in oocytes of younger patients, indicating gradual loss of cohesion or some other epigenetic alteration in the chromatin at centromeres (Duncan et al., 2012). Since there is no evidence that rates of aneuploidy increase in aged pig oocytes (Hornak et al., 2011), further studies on the role of the genetic background and the relative importance and ubiquitous loss of cohesion in aged oocytes are required.

\section{Unusal spindles, and synergistic roles of altered ex- pression patterns, permissive SAC and organelle dys- function in errors in chromosome segregation in aged mammalian oocytes}

Oocytes possess unusual spindles as multiple microtubule organizing centres (MTOCs) are present in ooplasm while centrioles disappear early in oogenesis. In mitosis of animal cells there is therefore an intrinsic bi-polarity established by replication of centrioles/centrosomes surrounded by pericentriolar material prior to M-phase. Disturbances in synchrony of replication of centrosomes in mitosis as for instance observed in cancer progression can contribute to high susceptibility to mitotic errors and chromosomal instability (CIN) (Thompson et al., 2010). In oocytes resuming maturation the multiple MTOCs form asters of microtubules all over the ooplasm. Upon germinal vesicle breakdown, microtubules from asters contact chromosome arms or interact with each other. Most MTOCs translocate to the centre of the cell forming a ball of microtubules in mouse oocytes. On the surface of this ball, bivalents align with arms of chromosomes parallel to the surface of the microtubular assemblies and not under tension from attachment of centromeres to microtubules (Kitajima et al., 2011). This stage is termed "circular bivalent" characterised by attachment of chromosome arms rather than centromeres to microtubules. Recent high resolution confocal microscopy of the behaviour of chromosomes and spindle formation in maturing mouse oocytes have shown that in a next prophase I stage the central microtubule ball rearranges to form a short bipolar spindle with very broad poles (Kitajima et al., 2011; Courtois et al., 2012). On this assembly the bivalents translocate to the outer middle, forming a hollow ring, a so-called "pre-prophase belt". Bivalents are connected by parallel attachments to the chromosome arms rather than attached kinetochores. Subsequently centromere attachment occurs, visible not only by microtubule tracing but also by chromosome stretching and initiation of bi-polar attachment of homologs in bivalents. During prometaphase I bivalents invade the spindle and oscillate between spindle poles, reorient and only finally align at the equator 
(chromosome congression). The initial attachment of centromeres to polar microtubules appears extremely error prone during this period. High resolution confocal microscopy suggests that individual centromeres re-attach to microtubules, the latter become de- and repolymerised and mono-or bipolarly attached such that about $90 \%$ of all chromosomes undergo one or more rounds of error correction on average in the mouse (Kitajima et al., 2011). Furthermore, centromeres of sister chromatids frequently attach to opposite spindle poles (amphitelic) instead of one spindle pole (syntelic) (Fig. 3 C,D,F), or one centromere becomes associated with spindle microtubules to both spindle poles (termed merotelic attachment) (Fig. 3G). If such irregular attachments are not resolved in time and remain unrecognized by the SAC, risks for chromosome lagging or separation of chromatids instead of homologs are increased. The correction of improper attachments involves microtubule depolymerases like Kif $2 \mathrm{~b}$ and Kif2c (also termed mitosis centromere associated kinesin, MCAK) and complex regulatory events that are controlling localisation and activity of the depolymerases, and thus microtubule turnover (reviewed by Eichenlaub-Ritter et al., 2010). Resolution of improper attachment aids chromosome congression at the equator and proper attachment and separation of chromosomes. It was only recently demonstrated that the SAC does not detect unaligned chromosomes in mammalian oocytes as long as chromosomes are attached to spindle fibres (Gui and Homer, 2012; Sebestova et al., 2012; Kolano et al., 2012). This makes oocytes uniquely susceptible to errors when amphitelic or merotelic and improper attachments persist to late metaphase I and into anaphase I.

The oocyte contains all elements for bipolar spindle assembly, even in absence of chromatin (Brunet et al., 1998). Depolymerisation of the spindle and even slight disturbances in polymerisation dynamics for instance by exposure of oocytes to low concentration of depolymerising drugs trigger meiotic arrest or delay when they occur in late prometaphase I and metaphase I stage once chromosomes become attached to the spindle by kinetochore fibres. The knockdown of SAC components causes precocious anaphase I progression showing that a SAC is functional in oocytes (reviewed by Homer et al., 2011). However, it is conceivable that the large size of the oocyte and the comparatively small spindle might contribute to relaxed SAC signalling. A critical mass of unattached chromosomes may be necessary to elicit signals preventing release from the SAC (Hoffmann et al., 2011). Furthermore, in cases where resolution of improper microtubule attachment is inefficient this is not sensed by the SAC since the SAC only recognizes attachment rather than position. Congression failure of chromosomes is thus much more frequent in aged compared to young oocytes (e.g. Battaglia et al., 1996) and is an indicator that controls may have been lost mediating proper centromere attachment and alignment of chromosomes. Furthermore, loss of steric constrains by deficiencies in the organization of centromeres may facilitate merotelic attachments. In fact, posttranslational modification of histones appeared altered in aged human metaphase II oocytes (Van den Berg et al., 2012) suggesting that epigenetic alterations of pericentromeric chromatin may be common.

\section{Relevance of altered expression in aged oocytes}

Since aneuploidy is low in oocytes of young females of most mammals, controls over chromosome segregation do not appear generally compromised. However, analysis of the transcriptome of aged metaphase II oocytes of mice and human detected consistent differences in transcript levels of genes involved in the SAC (e.g. Mad2, Bub1 and Msp1), the spindle, cell cycle control and in metabolism, particularly in mitochondrial function (Hamatani et al., 2004; Pan et al., 2007; Steuerwald et al., 2007; Grondahl et al., 2010). Although the alterations affected individual mRNAs differently, common pathways are involved that likely contribute to loss of control. For instance, some cell cycle components like TAp73, a member of the family of the p53 tumour suppressor protein family that declines with age in human oocytes (Steuerwald et al., 2007) is required for localisation of a SAC component. Alterations in its expression in aged oocytes (Hamatani et al., 2004; Grondahl et al., 2010; Guglielmino et al., 2012) can therefore also indirectly contribute to permissive SAC. Although it is still unknown to which extent altered transcript levels reflect altered proteome and enzyme activities or disturb regulation of the cytoskeleton and chromosome behaviour, under- or overexpression of individual SAC and spindle proteins might not cause a dramatic rise in errors in chromosome segregation, while the synergistic losses or gains and reduced availability of high energy substrates are much more likely to contribute to increased risks for meiotic errors in aged oocytes, particularly, when they contain susceptible chromosomes and such with reduced cohesion.

Expression in oogenesis is tightly and uniquely regulated. Oocytes are extremely large cells in the human body and have to supply the early embryo with transcripts, and protein to support development until zygotic gene activation- in humans at the 4-8 cell stage. Therefore, early embryogenesis relies on maternal gene products including ribosomes, tRNAs, mRNAs and microRNAs required for differential regulation of expression as well as proteins, membranes and cell organelles such as mitochondria, endoplasmic reticulum, and cortical granules (reviewed by Zuccotti et al., 2011). Studies in the mouse have shown that transcription increases dramatically when oocytes initiate the growth phase while fully grown, maturation and developmentally competent oocytes become transcriptonally silenced. Chromatin attains a so called "surrounded nucleolus" (SN) configuration within the GV of competent oocytes of most mammals including the human. When GV stage oocytes of mouse with a SN nuclear morphology are isolated from antral follicles past the time they are ready to resume maturation (preovulatory overmaturity) and are matured in vitro to metaphase II, they show increased aneuploidy compared to controls isolated from follicles at the appropriate time after stimulation of follicular development (unaged cohort) (Zuccotti et al., 1998). This suggests that prolonged transcriptional suppression and events prior to resumption of maturation may interfere with scheduled gene expression patterns, spindle formation and chromosome behaviour at maturation thus leading to meiotic errors.

Two independent studies using different sources and methods showed both significant differences in transcript abundance between oocytes from younger ( $<32$ years and $<32$ years) compared to aged ( $>40$ years and 37-39 years; respectively) women (Steuerwald et al.,2007; Grondahl et al., 2010). Age-related changes were prominent in the expression of genes involved in regulation and control of the cell-cycle, DNA damage response and repair, energy pathways, cytoskeletal structure and transcription. Transcripts in checkpoint control like Mad2, Bub1B and Bub3 were downregulated in aged mouse oocytes (Pan et al., 2007), as were transcripts in 
chromosome attachment and spindle regulation like MCAK and BRCA2. Knockdown or targeted knockout of these genes caused chromosome congression failure, spindle aberrations and aneuploidy (Pan et al., 2007). Interestingly, overall mRNA content appeared increased rather than decreased in aged human oocytes, and transcipts involved in the mitochondrial electron transport chain were more abundant. By contrast, those genes/transcripts related to ATP binding, oxidoreductase and the mitochondrial membrane appeared instead down-regulated in the oocytes from older women (Steuerwald et al., 2007). This could be a result of increased ROS, dysfunctional mitochondria and failures to stage-specifically recruit or degrade messages.

\section{Stimulation, environment during oocyte growth and maturation, and damage by ROS and AGEs may con- tribute to oocyte ageing and modulate susceptibility to meiotic errors}

Control of spindle formation, chromosome congression, chromosome separation, translation, intracellular signalling, and calcium homeostasis require availability of high energy substrates like ATP (reviewed by Eichenlaub-Ritter et al., 2011). During the growth phase somatic cell contacts contribute to the spatial organization and function of the meiotic spindle through actin-dependent mechanisms (e.g. Barrett and Albertini, 2010). Oocytes govern gene expression in the follicle by secreting growth factors which signal to the somatic compartment and direct metabolic activities. In turn, the cumulus supplies oocytes with metabolites like amino acids, lactate and pyruvate that are essential for maintaining mitochondrial activities, ATP production, and ion- and redox homeostasis in the oocyte (reviewed by Su et al., 2009). Oocytes contain large numbers of mitochondria but activity of mitochondria in meiotically arrested oocytes is comparatively low such that risks for oxidative damage by ROS are mimimal. Oocyte mitochondria contain few cristae, dense matrix and low inner membrane potential (reviewed by Eichenlaub-Ritter et al., 2010). Upon resumption of maturation a cortical domain of mitochondria with increased inner membrane potential is established upon loss of physical contacts between oocyte and cumulus. In vitro maturation of mouse oocytes is accompanied by stage-specific changes in distribution of mitochondria and bursts in ATP production (Yu et al., 2010). Loss of contacts via transzonal projection causes transient increases in inner membrane potential thus posing a risk for an untimely increased generation of ROS (e.g. Trapphoff et al., 2011). The accumulation of advanced glycation end products (AGEs) from reactive metabolites, e.g. from glycolysis, has been made responsible for age-related damage to mitochondria and membranes, increases in DNA breaks and adduct formation and senescence in many tissues (for references ses Tatone et al., 2011). Detoxifying enzymes appear less expressed in cumulus of aged compared to young mice, and exposures of mouse oocytes to methylglyoxal, one such reactive metabolite produced during glycolysis during in vitro maturation caused spindle aberrations and disturbances in chromosome congression in young mouse oocytes (Tatone et al., 2011). Exposure to low concentrations of methylglyoxal also interfered with the stage-specific accumulation of mitochondria in the vicinity of the spindle and affected the inner mitochondrial redox potential. Taken together with observations that obesity and diabetes increase spindle aberrations in young oocytes in mouse models (Wang et al., 2009), and that mice fed with moderately caloric restricted diet exhibit improved quality of aged oocytes and low aneuploidy rates (Selesniemi et al., 2010), all data point to a contributing role of alterations in follicular and oocyte metabolism and dysfunctional organelles in spindle aberrations and susceptibility to meiotic errors. In addition, mitochondria in aged human oocytes in resting primordial follicles and in follicle cells from stimulated cycles in ART are structurally and functionally altered (reviewed by Eichenlaub-Ritter et al., 2010). Depending on whether and to what extend such age-related alterations contribute to changes in follicular homeostasis, metabolism, altered expression patterns and loss of chromosome cohesion, it may become possible to prevent or delay these ageing events, e.g. by healthy life style (anti-oxidants in food, exercise, low fat diets etc.) or appropriate stimulation and handling in ART. In fact, there is evidence that milder stimulation decreases aneuploidy in human oocytes irrespective of maternal age (Baart et al., 2007). Whether high numbers of chromatids in aged human oocytes from stimulated cycles are originating from primary lesions like loss of cohesion due to age or are also partly related to sub-optimal intrafollicular environment in response to stimulation protocols, needs to be further investigated.

\section{Outlook}

In case the loss of chromosome cohesion, organelle dysfunction, and altered gene expression are inevitable events associated with oocyte ageing and the long meiotic arrest the possibility to improve aged oocyte quality may be restricted. However, when metabolism and follicular quality are influencing the status of chromatid cohesion, gene expression patterns, and spindle and cell cycle control, it may become feasible to delay oocyte ageing and reduce susceptibility to meiotic errors by preventive treatment and a healthy life style. With the advent of efficient and safe cryopreservation/vitrification protocols social freezing of young oocytes may become an option to prevent ageing, retain fertility and extend the reproductive period for women who want to postpone family-planning to more advanced ages. Research to generate oocytes from ovarian stem cells is promising to obtain more information on the basic cell biology of oogenesis and mechanisms behind oocyte ageing and eventually develop methods to preserve fertility to advanced ages. Currently, it appears imperative to raise awareness in young women and public of the still uncurable decay of oocyte quality and the risks for errors in chromosome segregation, associated with depletion of the follicle pool and the naturally limited reproductive period in humans.

\section{References}

ANGELL R (1997) First-meiotic-division nondisjunction in human oocyte. Am J Hum Genet 61: 23-32.

BAART EB, MARTINI E, EIJKEMANS MJ, et al., (2007) Milder ovarian stimulation for in-vitro fertilization reduces aneuploidy in the human preimplantation embryo: a randomized controlled trial. Hum Reprod 22: 980-988.

BAUDAT F, BUARD J, GREY C, et al., (2010) PRDM9 is a major determinant of meiotic recombination hotspots in humans and mice. Science 327: 836-840.

BARRETT SL, ALBERTINI DF (2010) Cumulus cell contact during oocyte maturation in mice regulates meiotic spindle positioning and enhances developmental competence. J Assist Reprod Genet 27: 29-39.

BATTAGLIA DE, GOODWIN P, KLEIN NA et al., (1996) Influence of maternal age on meiotic spindle assembly in oocytes from naturally cycling women. Hum Reprod 
11: 2217-2222.

BOND, J., CHANDLEY, A.C. (Ed.) (1983) Aneuploidy. Oxford University Press, Oxford. BROOK JD, GOSDEN RG, CHANDLEY AC. (1984) Maternal ageing and aneuploid embryos--evidence from the mouse that biological and not chronological age is the important influence. Hum Genet 66: 41-45.

BRUNET S, POLANSKI Z, VERLHAC MH, KUBIAK JZ, MARO B (1998) Bipolar meiotic spindle formation without chromatin. Curr Biol 8: 1231-1234

CHENG EY, HUNT PA, NALUAI-CECCHINI TA, et al., (2009) Meiotic recombination in human oocytes. PLoS Genet 5: e1000661.

CHIANG T, DUNCAN FE, SCHINDLER K, et al., (2010) Evidence that weakened centromere cohesion is a leading cause of age-related aneuploidy in oocytes. Curr Biol 20: 1522-1528.

CHIANG T, SCHULTZ RM, LAMPSON MA (2011) Age-dependent susceptibility of chromosome cohesion to premature separase activation in mouse oocytes. Biol Reprod 85: 1279-1283.

CUKURCAM S, BETZENDAHL I, MICHEL G, et al., (2007) Influence of follicular fluid meiosis-activating sterol on aneuploidy rate and precocious chromatid segregation in aged mouse oocytes. Hum Reprod 22: 815-828.

COURTOIS A, SCHUH M, ELLENBERG J, HIIRAGI T (2012) The transition from meiotic to mitotic spindle assembly is gradual during early mammalian development. J Cell Biol 198: 357-370.

DUNCAN FE, HORNICK JE, LAMPSON MA, et al., (2012) Chromosome cohesion decreases in human eggs with advanced maternal age. Aging Cell 11: 1121-1124.

EICHENLAUB-RITTER U, CHANDLEY AC, GOSDEN RG (1988) The CBA mouse as a model for age-related aneuploidy in man: studies of oocyte maturation, spindle formation and chromosome alignment during meiosis. Chromosoma 96:220-226.

EICHENLAUB-RITTER U, BOLL I (1989) Nocodazole sensitivity, age-related aneuploidy, and alterations in the cell cycle during maturation of mouse oocytes. Cytogenet Cell Genet 52: 170-176.

EICHENLAUB-RITTER U, STAUBACH N, TRAPPHOFF T (2010) Chromosomal and cytoplasmic context determines predisposition to maternal age-related aneuploidy: brief overview and update on MCAK in mammalian oocytes. Biochem Soc Trans 38: 1681-1686.

EICHENLAUB-RITTER U, WIECZOREK M, LÜKE S, et al., (2011) Age related changes in mitochondrial function and new approaches to study redox regulation in mammalian oocytes in response to age or maturation conditions. Mitochondrion 11: 783-796.

EMS-MCCLUNG SC, WALCZAK CE (2010) Kinesin-13s in mitosis: Key players in the spatial and temporal organization of spindle microtubules. Semin Cell Dev Biol 21: 276-282.

FRAGOULI E, ALFARAWATI S, GOODALL NN, SÁNCHEZ-GARCÍA JF, COLLS P, WELLS D (2011) The cytogenetics of polar bodies: insights into female meiosis and the diagnosis of aneuploidy. Mol Hum Reprod 17: 286-295.

FRAGOULI E, WELLS D (2011) Aneuploidy in the human blastocyst. Cytogenet Genome Res 133: 149-159.

GABRIELAS, THORNHILLAR, OTTOLINICS, etal., (2011)Array comparative genomic hybridisation on first polar bodies suggests that non-disjunction is not the predominant mechanism leading to aneuploidy in humans. JMed Genet 48: 433-427.

GARCIA-CRUZ R, BRIENO MA, ROIG I, et al., (2010) Dynamics of cohesin proteins REC8, STAG3, SMC1 beta and SMC3 are consistent with a role in sister chromatid cohesion during meiosis in human oocytes. Hum Reprod 25: 2316-2327.

GAULDEN ME (1989) A model that explains the varying frequency of aneuploid children with maternal age (J-shaped curve) as well as aneuploidy of paternal origin. Prog Clin Biol Res 318: 253-257.

GREY C, BARTHÈS P, CHAUVEAU-LE FRIEC G, LANGA F, BAUDAT F, DE MASSY B. (2011) Mouse PRDM9 DNA-binding specificity determines sites of histone $\mathrm{H} 3$ lysine 4 trimethylation for initiation of meiotic recombination. PLoSBio/9:e1001176.

GRØNDAHL ML, YDING ANDERSEN C, BOGSTAD J, et al., (2010) Gene expression profiles of single human mature oocytes in relation to age. Hum Reprod 25: 957-968.

GUGLIELMINO MR, SANTONOCITO M, VENTO M, et al., (2011) TAp73 is downregulated in oocytes from women of advanced reproductive age. Cell Cycle 10: 3253-3256.

GUI L, HOMER H (2012) Spindle assembly checkpoint signalling is uncoupled from chromosomal position in mouse oocytes. Development 139: 1941-1946.

HAADSMA ML, MOOIJ TM, GROEN H, et al., OMEGA Project Group. (2010) A reduced size of the ovarian follicle pool is associated with an increased risk of a trisomic pregnancy in IVF-treated women. Hum Reprod 25: 552-558.

HAMATANI T, FALCO G, CARTER MG, et al., (2004) Age-associated alteration of gene expression patterns in mouse oocytes. Hum Mol Genet 13: 2263-22678.

HANDYSIDEAH, MONTAG M, MAGLI MC, REPPING S, HARPER J, SCHMUTZLER A, VESELAK, GIANAROLI L, GERAEDTS J (2012) Multiple meiotic errors caused by predivision of chromatids in women of advanced maternal age undergoing in vitro fertilisation. Eur J Hum Genet 20: 742-747.

HARMAN D. (1956) Aging: a theory based on free radical and radiation chemistry. J Gerontol 11: 298-300.

HASSOLD TJ, JACOBS PA (1984) Trisomy in man. Annu Rev Genet 18: 69-97.

HASSOLD T, HALL H, HUNT P (2007) The origin of human aneuploidy: where we have been, where we are going. Hum Mol Genet 16(Spec No. 2):R203-R208.

HENDERSON SA, EDWARDS RG (1968) Chiasma frequency and maternal age in mammals. Nature 218: 22-28.

HIRSHFELD-CYTRON JE, DUNCANFE, XU M, JOZEFIKJK, SHEALD, WOODRUFF TK. (2011) Animal age, weight and estrus cycle stage impact the quality of in vitro grown follicles. Hum Reprod 26: 2473-2485

HODGES CA, REVENKOVA E, JESSBERGER R, et al., (2005) SMC1beta-deficient female mice provide evidence that cohesins are a missing link in age-related nondisjunction. Nat Genet 37: 1351-1355.

HOFFMANNS, MARO B, KUBIAKJZ, POLANSKIZ(2011) A single bivalent efficiently inhibits cyclin $B 1$ degradation and polar body extrusion in mouse oocytes indicating robust SAC during female meiosis I. PLoS One 6: e27143.

HOMER H (2011) New insights into the genetic regulation of homologue disjunction in mammalian oocytes. Cytogenet Genome Res 133: 209-22.

HORNAK M, JESETAM, MUSILOVAP, et al., (2011) Frequency of aneuploidy related to age in porcine oocytes. PLoS One 6:e18892.

HUNT PA, HASSOLD TJ. (2002) Sex matters in meiosis. Science 296: 2181-2183.

HUSSIN J, ROY-GAGNON MH, GENDRON R, et al., (2011) Age-dependent recombination rates in human pedigrees. PLoS Genet 7: e1002251.

ISHIGURO T, TANAKA K, SAKUNO T, et al., (2010) Shugoshin-PP2A counteracts casein-kinase-1-dependent cleavage of Rec8 by separase. Nat Cell Biol 12 500-506.

KITAJIMA TS, OHSUGI M, ELLENBERG J (2011) Complete kinetochore tracking reveals error-prone homologous chromosome biorientation in mammalian oocytes. Cell 146: 568-581.

KLINE JK, KINNEY AM, LEVIN B, et al., (2011) Trisomic pregnancy and elevated FSH: implications for the oocyte pool hypothesis. Hum Reprod 26: 1537-1550.

KONG A, THORLEIFSSON G, STEFANSSON H, et al., (2008) Sequence variants in the RNF212 gene associate with genome-wide recombination rate. Science 319: 1398-1401.

KONG A, BARNARD J, GUDBJARTSSON DF, et al., (2004) Recombination rate and reproductive success in humans. Nat Genet 36: 1203-1206.

KOLANO A, BRUNET S, SILK AD, CLEVELAND DW, VERLHAC MH (2012) Errorprone mammalian female meiosis from silencing the spindle assembly checkpoint without normal interkinetochore tension. Proc NatlAcad SciUSA 109: E1858-1867.

KOUZNETSOVA A, LISTER L, NORDENSKJOLD M, et al.(2007) Bi-orientation of achiasmatic chromosomes in meiosis I oocytes contributes to aneuploidy in mice. Nat Genet 39: 966-968.

KURAHASHI H, KOGO H, TSUTSUMI M, INAGAKI H, OHYE T. (2012) and sexspecific reproduction problems. Front Genet 3: 112.

LANE SI, YUNY, JONES KT (2012) Timing of anaphase-promoting complex activation in mouse oocytes is predicted by microtubule-kinetochore attachment but not by bivalent alignment or tension. Development 139: 1947-1955

LEMAIRE-ADKINS R, RADKE K, HUNT PA. (1997) Lack of checkpoint control at the metaphase/anaphase transition: a mechanism of meiotic nondisjunction in mammalian females. J Cell Biol 139: 1611-1619.

LISTER LM, KOUZNETSOVA A, HYSLOP LA, et al., (2010) Age-related meiotic segregation errors in mammalian oocytes are preceded by depletion of cohesin and Sgo2. Curr Biol 20: 1511-1521.

MAGLI MC, GRUGNETTIC, CASTELLETTIE, PAVIGLIANITIB, FERRARETTIAP, et al., (2012) Five chromosome segregation in polar bodies and the corresponding oocyte. Reprod Biomed Online 24: 331-338. 
MAILHES JB, YOUNG D, LONDON SN (1998) Postovulatory ageing of mouse oocytes in vivo and premature centromere separation and aneuploidy. Biol Reprod 58: 1206-1210.

MERRIMAN JA, JENNINGS PC, MCLAUGHLIN EA, et al., (2012) Effect of aging on superovulation efficiency, aneuploidy rates, and sister chromatid cohesion in mice aged up to 15 months. Biol Reprod 86: 49, 1-6.

MUSACCHIO A (2011) Spindle assembly checkpoint: the third decade. Philos Trans $R$ Soc Lond B Biol Sci 366: 3595-3604.

NAGAOKA SI, HODGES CA, ALBERTINI DF, et al., (2011) Oocyte-specific differences in cell-cycle control create an innate susceptibility to meiotic errors. Curr Biol 21: 651-657.

NAGAOKA SI, HASSOLD TJ, HUNT PA (2012) Human aneuploidy: mechanisms and new insights into an age-old problem. Nat Rev Genet 13: 493-504.

NASMYTH K (2011) Cohesin: a catenase with separate entry and exit gates? Nat Cell Biol 13: 1170-7.

OH JS, SUSOR A, CONTI M (2011) Protein tyrosine kinase Wee1B is essential for metaphase II exit in mouse oocytes. Science 332: 462-465.

OLIVER TR, FEINGOLD E, YU K, et al., (2008) New insights into human nondisjunction of chromosome 21 in oocytes. PLoS Genet 4: e1000033.

PACCHIEROTTIF, ADLER ID, EICHENLAUB-RITTERU, etal., (2007) Gender effects on the incidence of aneuploidy in mammalian germ cells. Environ Res 104: 46-69.

PACCHIEROTTIF, EICHENLAUB-RITTERU. (2011) Environmental hazard in the aetiology of somatic and germ cell aneuploidy. Cytogenet Genome Res 133:254-268.

PAN H, O'BRIEN MJ, WIGGLESWORTH K, et al., (2005) Transcript profiling during mouse oocyte development and the effect of gonadotropin priming and development in vitro. Dev Biol 286: 493-506.

PELLESTOR F, ANDRÉO B, ARNAL F, et al., (2003) Maternal aging and chromosomal abnormalities: new data drawn from in vitro unfertilized human oocytes. Hum Genet 112: 195-203.

PENROSE LS (1933) The relative effects of paternal and maternal age in mongolism. J Genet 27: 219-224.

REVENKOVAE, HERRMANN K, ADELFALK C, et al., (2010) Oocyte cohesin expression restricted to predictyate stages provides full fertility and prevents aneuploidy. Curr Biol 20: 1529-1533.

ROBINSON WP, KUCHINKABD, BERNASCONI F, PETERSEN MB, SCHULZE A, et al., (1998) Maternal meiosis I non-disjunction of chromosome 15: dependence of the maternal age effect on level of recombination. Hum Mol Genet 7: 1011-1019.

SEBESTOVA J, DANYLEVSKA A, DOBRUCKA L, KUBELKA M, ANGER M (2012) Lack of response to unaligned chromosomes in mammalian female gametes. Cell Cycle 11: 3011-3018

SELESNIEMI K, LEE HJ, TILLY JL (2008) Moderate caloric restriction initiated in rodents during adulthood sustains function of the female reproductive axis into advanced chronological age. Aging Cell 7: 622-629.

SHIN YH, CHOI Y, ERDIN SU, et al., (2010) Hormad1 mutation disrupts synaptonemal complex formation, recombination, and chromosome segregation in mammalian meiosis. PLoS Genet 6: e1001190.

SMITZJ, PICTON HM, PLATTEAU P, et al.,2007) Principal findings from a multicenter trial investigating the safety of follicular-fluid meiosis-activating sterol for in vitro maturation of human cumulus-enclosed oocytes. Fertil Steril 87: 949-964.

SOEWARTO D, SCHMIADY H, EICHENLAUB-RITTER U (1995) Consequences of non-extrusion of the first polar body and control of the sequential segregation of homologues and chromatids in mammalian oocytes. Hum Reprod 10: 2350-2360.

STEUERWALD NM, BERMÚDEZ MG, WELLS D, et al., (2007) Maternal age-related differential global expression profiles observed in human oocytes. Reprod Biomed Online 14: 700-708.
STOLK L, PERRY JR, CHASMAN DI, et al.,2012) Meta-analyses identify 13 loci associated with age at menopause and highlight DNA repair and immune pathways. Nat Genet 44: 260-268.

SU YQ, SUGIURA K, EPPIG J (2009) Mouse oocyte control of granulosa cell development and function: paracrine regulation of cumulus cell metabolism. Semin Reprod Med 27: 32-42.

SUSIARJO M, HASSOLD TJ, FREEMAN E, HUNT PA (2007) Bisphenol A exposure in utero disrupts early oogenesis in the mouse. PLoS Genet 3: e5.

TACHIBANA-KONWALSKI K, GODWIN J, VAN DER WEYDEN L, et al., (2010) Rec8-containing cohesin maintains bivalents without turnover during the growing phase of mouse oocytes. Genes Dev 24: 2505-2516.

TANNOY, KITAJIMATS, HONDAT, et al., (2010) Phosphorylation of mammalian Sgo2 by Aurora B recruits PP2A and MCAK to centromeres. Genes Dev 24:2169-2179.

TATONE C, HEIZENRIEDER T, DI EMIDIO G, et al., (2011) Evidence that carbonyl stress by methylglyoxal exposure induces DNA damage and spindle aberrations, affects mitochondrial integrity in mammalian oocytes and contributes to oocyte ageing. Hum Reprod 26: 1843-1859.

THOMPSON SL, BAKHOUM SF, COMPTONDA(2010) Mechanisms of chromosomal instability. Curr Biol 20: R285-295.

TRAPPHOFF T, EL HAJJ N, ZECHNER U, HAAF T, EICHENLAUB-RITTER U (2010) DNA integrity, growth pattern, spindle formation, chromosomal constitution and imprinting patterns of mouse oocytes from vitrified pre-antral follicles. Hum Reprod 25: 3025-3042.

VAN DEN BERG IM, ELEVELD C, VAN DER HOEVEN M, et al., (2011) Defective deacetylation of histone $4 \mathrm{~K} 12$ in human oocytes is associated with advanced maternal age and chromosome misalignment. Hum Reprod 26: 1181-1190.

VAN DER STROOM EM, KÖNIG TE, VAN DULMEN-DEN BROEDER E, et al., (2011) Early menopause in mothers of children with Down syndrome? Fertil Steril 96: 985-990.

VOGT E, KIRSCH-VOLDERS M, PARRY J, et al., (2008) Spindle formation, chromosome segregation and the spindle checkpoint in mammalian oocytes and susceptibility to meiotic error. Mutat Res 651: 14-29.

VOLARCIK K, SHEEAN L, GOLDFARB J, et al., (1998) The meiotic competence of in-vitro matured human oocytes is influenced by donor age: evidence that folliculogenesis is compromised in the reproductively aged ovary. Hum Reprod 13: 154-160.

WANG Q, RATCHFORD AM, CHI MM, et al., (2009) Maternal diabetes causes mitochondrial dysfunction and meiotic defects in murine oocytes. Mol Endocrinol 23: 1603-1612.

WHITE YA, WOODS DC, TAKAI Y, ISHIHARA O, SEKI H, TILLY JL. (2012) Oocyte formation by mitotically active germ cells purified from ovaries of reproductive-age women. Nat Med 18: 413-421.

XU YW, PENG YT, WANG B, ZENG YH, ZHUANG GL, ZHOU CQ (2011) High follicle-stimulating hormone increases aneuploidy in human oocytes matured in vitro. Fertil Steril 95: 99-104.

YU Y, DUMOLLARD R, ROSSBACH A, et al., (2010) Redistribution of mitochondria leads to bursts of ATP production during spontaneous mouse oocyte maturation. J Cell Physiol 224: 672-680.

ZUCCOTTI M, BOIANI M, GARAGNA S, REDI CA (1998) Analysis of aneuploidy rate in antral and ovulated mouse oocytes during female aging. Mol Reprod Dev 50: 305-312.

ZUCCOTTI M, MERICO V, CECCONI S, REDI CA, GARAGNA S (2011) What does it take to make a developmentally competent mammalian egg? Hum Reprod Update 17: 525-540. 


\section{Further Related Reading, published previously in the Int. J. Dev. Biol.}

Germline stem cells and sex determination in Hydra

Chiemi Nishimiya-Fujisawa and Satoru Kobayashi

Int. J. Dev. Biol. (2012) 56: 499-508

The Dr-nanos gene is essential for germ cell specification in the planarian Dugesia ryukyuensis Haruka Nakagawa, Hirotsugu Ishizu, Ayako Chinone,Kazuya Kobayashi and Midori Matsumoto Int. J. Dev. Biol. (2012) 56: 165-171

Impaired meiotic competence in putative primordial germ cells produced from mouse embryonic stem cells

Marianna Tedesco, Donatella Farini and Massimo De Felici

Int. J. Dev. Biol. (2011) 55: 215-222

A polymorphic, thrombospondin domain-containing lectin is an oocyte marker in Hydractinia: implications for germ cell specification and sex determination

Brahim Mali, R. Cathriona Millane, Günter Plickert, Marcus Frohme and Uri Frank Int. J. Dev. Biol. (2011) 55: 103-108

Generation of germ-line chimera zebrafish using primordial germ cells isolated from cultured blastomeres and cryopreserved embryoids

Yutaka Kawakami, Rie Goto-Kazeto, Taiju Saito, Takafumi Fujimoto, Shogo Higaki, Yoshiyuki Takahashi, Katsutoshi Arai and Etsuro Yamaha

Int. J. Dev. Biol. (2010) 54: 1493-1501

In vitro germ cell differentiation during sex differentiation in a teleost fish

Tohru Kobayashi

Int. J. Dev. Biol. (2010) 54: 105-111

5 yr ISI Impact Factor $(2011)=2.959$

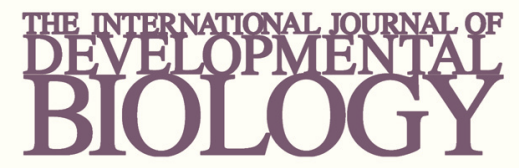

Volume 54 Nos. $6 / 7$
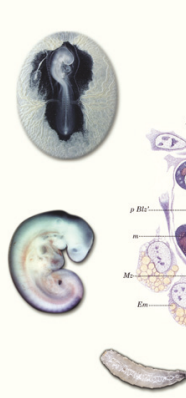

Developmental Hematopoiesis
Special Issue

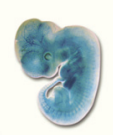

(2) (8)

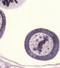

(1)
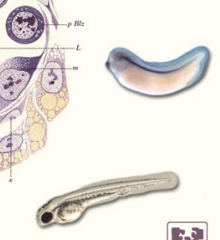

5

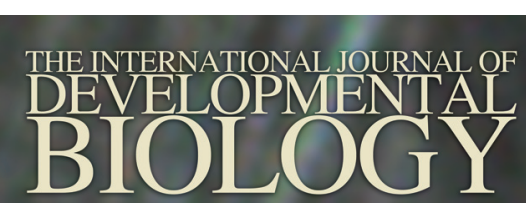

Volume 56 Nos. 1/2/3

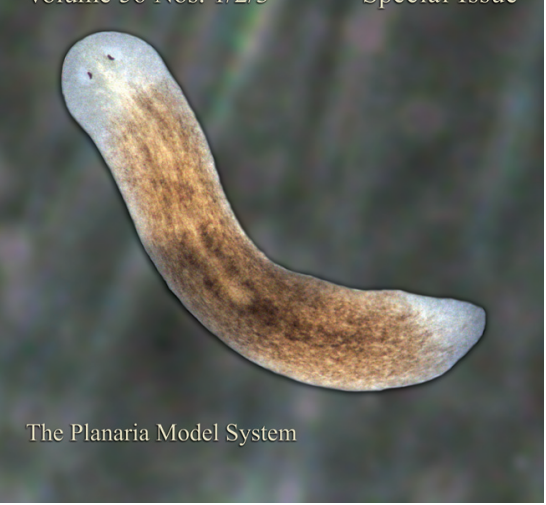

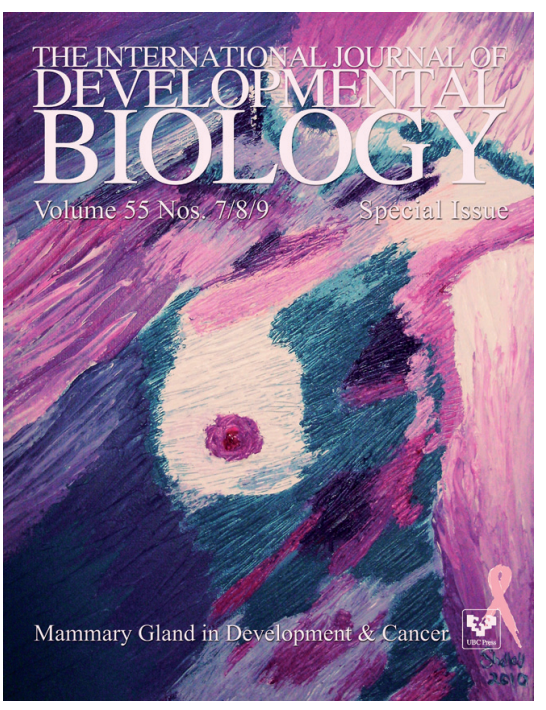

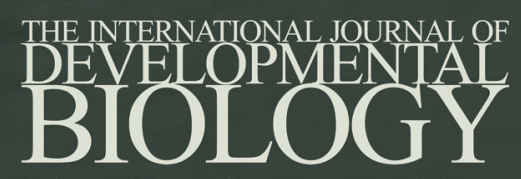

Volume 55 Nos. 4/5

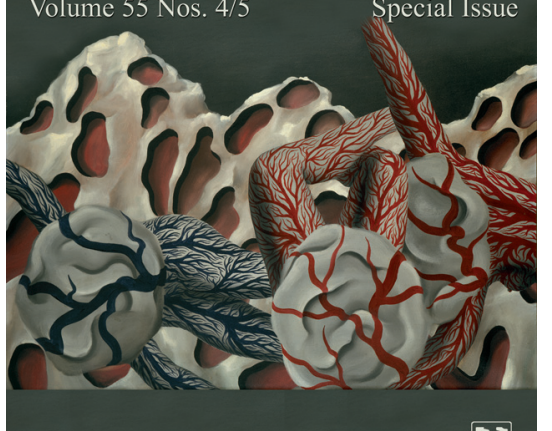

Angiogenesis in Development \& Cancer

政 